\title{
Effects of induced dysphoric mood on fact retrieval and inferential reasoning from world knowledge
}

\author{
CAMERON J. CAMP \\ University of New Orleans, New Orleans, Louisiana \\ and \\ MICHAEL F. PIGNATIELLO \\ Preble County Counseling Center, Eaton, Ohio
}

\begin{abstract}
World knowledge stimuli were used to investigate the effects of an induced dysphoric mood on fact retrieval and inferential reasoning processes. Sixty young adults were divided randomly into three mood groups (elated, neutral, and dysphoric). The subjects in each group read a different set of 30 sentences, each set designed to induce a specific mood. Two world knowledge question types were then presented, one designed to induce fact retrieval alone and the other designed to induce inferential reasoning. Significant differences for question type were found, with inference questions requiring longer response times and being rated as involving more complex processing than fact retrieval questions. However, groups were not significantly different for number of correct responses, confidence levels, introspective process ratings, or speed of response for either type of question. Thus, induced dysphoric mood did not produce a significant negative impact on utilization of world knowledge.
\end{abstract}

The focus of this research was to determine whether an induced dysphoric mood would impair utilization of information from world knowledge, knowledge acquired through life experiences by adults. (Unless clinical populations were used, we will use the term dysphoria rather than depression in describing our research and the research of others who have studied nonclinical populations.) Ellis and his colleagues (Ellis, Thomas, McFarland, \& Lane, 1985; Ellis, Thomas, \& Rodriguez, 1984; Leight \& Ellis, 1981; Tyler, Hertel, McCallum, \& Ellis, 1979) have demonstrated that inducing a dysphoric mood in normal subjects impairs retention of new information. This effect was found when the dysphoric mood was induced either before encoding new material or after encoding but before attempted recall. However, impaired memory was not found for recognition tasks when dysphoria was induced (Ellis, personal communication, 1984).

Ellis (1985; Ellis \& Ashbrook, 1988) points out that studies that do not show memory deficits associated with depression may have utilized tasks that do not require large amounts of processing capacity or cognitive effort. Weingartner and his colleagues (Cohen, Weingartner, Smallberg, Pickar, \& Murphy, 1982; Weingartner, 1986;

This research was supported by a grant from the Graduate Research Council of the University of New Orleans, awarded to C.J.C. Correspondence regarding this manuscript should be addressed to C. J. Camp, Department of Psychology, University of New Orleans, New Orleans, LA 70148 (cjcps@uno.bitnet).
Weingartner, Miller, \& Murphy, 1977) also have reported that depressives show memory performance deficits when effortful information processing was required, but not when automatic information processes were used.

Niederehe (1986) compared younger and older adult depressives from an outpatient population with agematched controls. Niederehe found no effects of depression on both memory for a newly learned list of words and accuracy in answering factual questions based on world knowledge. These findings were true for free recall, cued recall, and recognition for both types of memory tasks. He also noted that the tasks he used may not have required high enough levels of cognitive effort for a depression-related memory deficit to have been found.

The present study was designed to determine whether an induced dysphoric mood could produce deficits in the use of world knowledge (i.e., tertiary or remote memory). This was done by varying the amount of cognitive effort needed to answer questions utilizing information from world knowledge. Camp and his colleagues (Camp, 1981, 1989; Camp, Lachman, \& Lachman, 1980; Camp \& Pignatiello, 1988) developed world knowledge questions designed to initiate two question-answering processes-fact retrieval and inferential reasoning. An example of a fact retrieval question would be, "What was the name of the first satellite orbited by the Russians?" "'Sputnik." An example of an inference question would be, "What piece of sporting goods equipment would a teller at a drive-in bank find most useful?"'- "tennis ball can." Since inferential reasoning is related to longer response times than fact retrieval (and involves the cre- 
ation of a new association), it was assumed that inferential reasoning requires more cognitive effort than fact retrieval does. If this is so, inferential reasoning might be more impaired by an induced dysphoric mood than would fact retrieval from world knowledge systems.

Camp and Pignatiello (1988) have described a study in which older and younger adults were presented fact retrieval and inference questions followed by a single alternative (true/false task) or four alternatives (multiplechoice task). Older adults either outperformed or were equal to younger adults in all cases except for inference questions presented in a multiple-choice format. In that case, older adults were significantly less accurate than younger adults. Furthermore, all age groups took longest in answering inference questions in a multiple-choice format, thus providing more support for the contention that inference questions in this format maximized the cognitive effort necessary to answer world knowledge questions.

Consequently, in this study, fact retrieval and inference questions were presented in a multiple-choice format to individuals in induced mood states. Accuracy of responses, speed of responses, and confidence in responses were measured for both types of questions. It was hypothesized that measures associated with inferential reasoning would be more adversely affected by an induced dysphoric mood than would measures associated with fact retrieval.

\section{METHOD}

\section{Subjects}

The experimental sample comprised 60 young adults ( 30 males and 30 females) enrolled in psychology courses at the University of New Orleans. Equal numbers of males and females were randomly assigned to each of three experimental groups-dysphoric, neutral, and elated. All participants were screened for symptoms of depression by using the Beck Depression Inventory (BDI), short form. An exclusion criterion of 14 points or more was set, and no participants scored within the exclusion range. An analysis of variance (ANOVA) indicated that the three experimental groups also were not significantly different on this measure $[F(2,57)=2.21, p>.10]$. Means (and $S D s)$ for the dysphoric, neutral, and elated groups were 4.1 (3.2), 3.1 (2.9), and 2.2 (2.1), respectively.

\section{Materials}

World knowledge questions. Thirty questions were chosen from a larger pool of questions used in a previous study (Camp \& Pignatiello, 1988) and were presented to the participants. Fifteen questions were intended to initiate fact retrieval, and 15 were intended to initiate inferential reasoning. The two question types were matched for level of difficulty, and order of presentation was randomized for each participant.

Of the four alternatives listed for each question, one answer was correct, one sounded like the correct answer, one was related semantically to the correct answer, and one sounded like the semantically related false alternative. For example, the four choices for the question "What is the capital of Lebanon?"' were: "Beirut," "Birren," "Damascus," and "Demetas."

Following the selection of an answer, the participant indicated on a 3-point scale the type of reasoning process used in answering the question: 1 = mostly "remembered"; 2 = mostly eliminated alternatives; 3 = mostly "figured out" the response. The subjects then rated their confidence in their response on a 4-point scale: 1 = certainly right; 2 = probably right; 3 = probably wrong; $4=$ random choice .

Mood induction. A modification of Velten's (1968) procedure for mood induction was used. In the typical Velten procedure, subjects read
60 sentences, each typed on a separate card. One group of participants reads 60 dysphoric statements, such as "Every now and then I feel so tired and gloomy that I'd rather just sit than do anything." Another group reads 60 elated statements, such as "If your attitude is good, then things are good, and my attitude is good." A final group reads 60 neutral statements, such as "The filter allows rays of its own color to pass through." We used the same procedure, except that we presented 30 sentences in each condition instead of 60 . This format was chosen because Ellis and his colleagues have found that $\mathbf{3 0}$ sentences are just as effective as 60 sentences (Ellis et al., 1985). We differed from Ellis and his colleagues in one respect. They had participants read preparatory statements before the mood induction procedure and asked the participants to "talk themselves into the mood." However, since these preparatory statements may have created potentially high demand characteristics (Buchwald, Strack, \& Coyne, 1981; Polivy \& Doyle, 1980), we simply had the participants read the mood induction sentences aloud without using preparatory statements. The participants were paced so that they proceeded to the next card every $20 \mathrm{sec}$.

In order to assess mood state, the participants were asked to rate their moods immediately following the mood induction task. Mood was rated by using the short form of the Depression Adjective Check List (DACL), which required the participants to rate how accurately a series of 14 adjectives described their current mood (i.e. "not at all," " a little bit," or "a lot"). The DACL contained eight adjectives describing a negative feeling and six adjectives describing a positive feeling. The point values assigned to the negative items were 0,2 , and 4 points for responses marked "not at all," "a little bit," or "a lot," respectively. The point values for the positive items were 2,1 , and 0 points, respectively.

\section{Procedure}

The participants first completed 8 practice questions dealing with world knowledge to familiarize themselves with the question answering procedures. Next, they were given the mood induction procedure, followed by the administration of the DACL, and then the presentation of the 30 experimental questions. The procedures used for the 30 questions were the same as those described for the practice questions.

For question answering, a question would appear on a computer monitor. After reading the question, the participant pressed the space bar of a microcomputer keyboard, which triggered the four alternatives to appear on the monitor. After deciding on an answer, the participant pressed the space bar again, which caused the response time for answer selection (time between the first press of the space bar and the second press of the space bar) to be recorded. The participant then verbalized his or her selection to the experimenter, who entered the selection into the microcomputer on a separate keyboard. Next, the subject indicated the reasoning process that was used when he/she answered the question (3-point rating scale) and the degree of confidence in the response (4-point rating scale). These ratings were recorded by the experimenter as previously described.

\section{RESULTS}

\section{Mood Assessment Measure}

The mean scores (and SDs) on the DACL for the dysphoric, neutral, and elated conditions were $13.8(6.5)$, 10.4 (6.0), and 8.2 (4.4), respectively. The three mood conditions produced a significant difference for this measure $[F(2,57)=5.1, p<.05]$, with the means aligning themselves in the hypothesized direction. Tukey $H S D$ comparisons revealed that the mean of the dysphoric group was significantly higher than that of the elated group. No other comparisons were significant. (This pattern also has been found when DACL scores are taken after musical passages are used to induce these moods; see Pignatiello, Camp, \& Rasar, 1986, Experiments 1 and 2). 


\section{World Knowledge Questions}

The data associated with world knowledge questions were analyzed with $3 \times 2$ mixed model ANOVAs. The three levels of mood condition served as the betweensubject factor and the two levels of question type (fact retrieval and inference) served as the within-subject factor. Only correct responses that were not selected randomly (i.e., responses not associated with a confidence rating of "random choice") were used in these analyses. This stipulation was made since past research (e.g., Camp et al., 1980) has demonstrated that randomly selected answers may be associated with unintended or unknown selection processes.

Correct responses. The analysis for the number of nonrandom correct responses revealed no significant effects. All mood conditions displayed equal levels of accuracy across both question types. The mean numbers of nonrandom correct responses (and $S D$ s) for fact retrieval and inference questions were 10.8 (2.5) and $10.5(2.4)$, respectively. The ANOVA results for the effects of group and question type and for the group $\times$ question type interaction were $F(2,57)=0.24, p>.50 ; F(1,57)=0.90$, $p>.30$; and $F(2,57)=2.48, p>.08$, respectively.

Confidence ratings. No effects were significant for this measure. Mean ratings (and $S D$ s) for the fact retrieval and inference questions were $1.8(.4)$ and $1.7(.4)$, respectively. The ANOVA results for the effects of group and question type and for the group $\times$ question type interaction were $F(2,57)=1.21, p>.30 ; F(1,57)=0.85$, $p>.30$; and $F(2,57)=2.37, p>.10$, respectively.

Response speed. Reaction times (RTs) for selecting answers to questions underwent reciprocal transformations so that a normal distribution could be imposed on these data. The average reciprocal RT of correct, nonrandom responses for each subject for each question type were the data used in these analyses. These RTs yielded a significant effect for question type $[F(1,57)=103$, $p<.01$ ]. Fact retrieval questions were answered more swiftly than inference questions, replicating an effect found in previous studies (Camp, 1981; Camp et al., 1980; Camp \& Pignatiello, 1988). Mean reciprocal RTs (and $S D$ s) for fact retrieval and inference questions were $24.5(8.3)$ and 15.5 (4.9), respectively. No other effects were significant. The ANOVA results for the effect of group and the group $\times$ question type interaction were $F(2,57)=0.38, p>.60 ;$ and $F(2,57)=0.93$, $p>.40$, respectively.

Reasoning process ratings. Introspective ratings of the processes used to answer questions were analyzed next. A significant effect of question type emerged $[F(1,57)=$ $135, p<.01$ ]. Mean ratings (and $S D$ s) for fact retrieval and inference questions were $1.5(.3)$ and $2.3(.4)$, respectively. Fact retrieval questions were associated with ratings that indicated significantly less inferential reasoning than ratings for inference questions. No other effects were significant. The ANOVA results for the effect of group and for the group $\times$ question type interaction were $F(2,57)=1.39, p>.20 ;$ and $F(2,57)=0.30$, $p>.70$, respectively.

\section{Summary of World Knowledge Questions' Results}

Taken together, the RTs and process ratings data indicated that the two question types elicited the intended differential processes. Mood condition did not influence these measures and did not interact with question type. Given that the fact retrieval and inference questions were matched for level of difficulty before the experiment was performed, the lack of question type effects for number of correct responses and confidence ratings was not surprising.

\section{Exploratory Analyses}

Comparisons between dysphoric and elated groups. Since the post hoc analyses had indicated that only the dysphoric and elated groups were different significantly from each other on the DACL measure, a series of $2 \times 2$ ANOVAs were conducted to compare the dysphoric and elated groups, with question type used as a within-subject factor. The same pattern of outcomes as that described above was found for world knowledge measures.

Gender effects. The four world knowledge measures were reanalyzed with a 3 (group) $\times 2$ (gender) $\times 2$ (question type) mixed model ANOVA. There was a main effect of gender for confidence ratings $[F(1,54)=5.19$, $p<.027$ ]. Mean levels of confidence (and $S D$ s) for the women and men were $1.59(.3)$ and $1.77(.3)$, respectively. Women were slightly more confident than men in their overall answers. There was also a group $\times$ gender $X$ question type interaction for the reciprocal reaction time data $[F(2,54)=4.35, p<.02]$. No other effects associated with gender were significant.

Correlational analyses. The BDI and DACL measures were correlated both with scores for all of the world knowledge measures for each question type and with sex, using Pearson product moment correlation coefficients (rs). For all of these correlations, only two were significant at the $p<.05$ level. Confidence ratings for inference questions were correlated with both the BDI ( $r=$ $.34)$ and the DACL $(r=.25)$ measures. This indicated that an increased level of dysphoria as measured by these two scales was related to a decreased confidence in answers associated with inferential reasoning.

\section{DISCUSSION}

Past research outcomes have shown that dysphoria may influence cognition, especially memory, if cognitive effort must be expended for one to perform well. This has been found in both clinical populations and college populations, in studies done with mood induction. Though one might argue that this is primarily an encoding issue, Ellis et al. (1985) have demonstrated that dysphoric mood induction after learning, but before recall, may inhibit recall performance. Still, most of this research has involved the use of tests for newly learned material.

Our results coincided with other studies demonstrating that answering real-world knowledge questions does not seem to be affected adversely by factors such as age or depression. This type of information, possessing a high degree of ecological validity, seems to remain accessible under a variety of adverse conditions. Maintaining the integrity of such a knowledge base is a highly adaptive function for our species. Therefore, it is not too surprising that access to such knowledge is impaired in only the most extreme circumstances, such as closed head injuries or progressive dementia. Though recall failures such as tip-of- 
the-tongue states are common in normal adults, recognition memory for such information is consistently high.

The correlation between dysphoria and confidence in answers for inference questions, though small, is intriguing. If this effect proves reliable, it will be interesting to determine whether similar (perhaps larger) effects might be found in clinical populations, in normal populations by using different types of mood induction procedures, or in less constrained retrieval tasks. It also would be useful to determine whether this measure is related more to measures of memory complaint than memory per se (see Niederehe, 1986, for a discussion of the relationship between memory complaint vs. memory impairment in depressives). Nevertheless, at this point we support the position that the utilization of world knowledge in a recognition memory format, even under conditions of increased cognitive effort, does not seem to be affected by induced dysphoric mood.

\section{REFERENCES}

Buchwald, A. M., Strack, S., \& Coyne, J. C. (1981). Demand characteristics in the Velten mood induction procedure. Journal of Consulting \& Clinical Psychology, 49, 478-479.

CAMP, C. J. (1981). The use of fact retrieval versus inference in young and elderly adults. Journal of Gerontology, 36, 715-721.

CAMP, C. J. (1989). World knowledge systems. In L. Poon, D. Reubens, \& B. Wilson (Eds.), Everyday cognition in adult and late life (pp. 457-482). New York: Cambridge University Press.

Camp, C. J., Lachman, J. L., \& Lachman, R. (1980). Evidence for direct access and inferential retrieval in question answering. Journal of Verbal Learning \& Verbal Behavior, 19, 583-596.

Camp, C. J., \& Pignatiello, M. F. (1988). Beliefs about fact retrieval and inferential reasoning across the adult lifespan. Experimental Aging Research, 14, 89-97.

Cohen, R. M., Weingartner, H., Smallberg, S. A., Pickar, D., \& MURPHY, D. L. (1982). Effort and cognition in depression. Archives of General Psychiatry, 39, 593-597.

Ellis, H. C. (1985). On the importance of mood intensity and encoding demands in memory: Commentary on Hasher, Rose, Zacks, Sanft, and Doren. Journal of Experimental Psychology: General, 114, 392-395.

Ellis, H. C., \& AshBrook, P. W. (1988). Resource allocation model of the effects of depressed mood state on memory. In K. Fiedler \& J. Forgas (Eds.), Affect, cognition, and social behavior (pp. 25-43). Toronto: Hogrefe.

Ellis, H. C., Thomas, R. L., McFarland, A. D., \& Lane, J. W. (1985). Emotional mood states and retrieval in episodic memory. Journal of Experimental Psychology: Learning, Memory, \& Cognition, 11, 363-370.

Eluis, H. C., Thomas, R. L., \& Rodriguez, I. A. (1984). Emotional mood states and memory: Elaborative encoding, semantic processing, and cognitive effort. Journal of Experimental Psychology: Learning, Memory, \& Cognition, 10, 470-472.

LEIGHT, K. A., \& Ellis, H. C. (1981). Emotional mood states, strategies, and state dependency in memory. Journal of Verbal Learning \& Verbal Behavior, 20, 251-266.

Niederehe, G. (1986). Depression and memory impairment in the aged. In L. Poon (Ed.), Handbook for clinical memory assessment of older adults (pp. 226-237). Washington, DC: American Psychological Association.

Pignatiello, M. F., Camp, C. J., \& Rasar, L. A. (1986). Musical mood induction: An alternative to the Velten technique. Journal of Abnormal Psychology, 95, 295-297.

Polivy, J., \& DoYle, C. (1980). Laboratory induction of mood states through the reading of self-referent mood statements: Affective changes or demand characteristics? Journal of Abnormal Psychology, 89, 286-290.

Tyler, S. W., Hertel, P. T., McCallum, M. C., \& Ellis, H. C. (1979). Cognitive effort and memory. Journal of Experimental Psychology: Human Learning \& Memory, 5, 607-617.

VELTEN, E. (1968). A laboratory task for the induction of mood states. Behavioral Research \& Therapy, 6, 474-482.

WeINGARTNER, H. (1986). Automatic and effort-demanding cognitive processes in depression. In L. Poon (Ed.), Handbook for clinical memory assessment of older adults (pp. 218-225). Washington, DC: American Psychological Association.

Weingartner, H., Miller, H., \& MURPhy, D. L. (1977). Mood-statedependent retrieval of verbal associations. Journal of Abnormal Psychology, 86, 276-284.

(Manuscript received June 24, 1992.) 